\title{
FORMAÇÃo DO HERBÁRIO DE CARAJÁS - HCJS
}

\author{
Luís Carlos Nepomuceno da Silva ${ }^{1}$ \\ Gisele da Silva Staudohar \\ Carlindo Milhomem de Araújo
}

\begin{abstract}
RESUMO - Descreve-se a formação e o conteúdo do Herbário de Carajás, sediado no Parque Botânico de Carajás, km 85 PA-275, com funcionamento a partir de fevereiro de 1987 e cuja área de abrangência é a Serra dos Carajás. A montagem do Herbário envolveu investiònentos em reformas prediais, compra de material e climatização do espaço físico, recebendo-se doações de alguns equipamentos pelo PNUD. O treinamento de pessoal em coletas e preparo de exsicatas foi totalmente realizado em Carajás. A coleta de material fértil é realizada nos ecossistemas existentes: campos rupestres, área de transição e floresta. A identificação a nível de família Botânica é realizada no próprio laboratório do Parque e a metodologia em implantação é o envio de material botânico para especialistas de cada familia de diferentes instituições, interessados no recebimento deste material. O Herbário vem atingindo seu objetivo principal que é coexistir, dentro de um programa de estudos ambientais, com a atividade mineradora, fomentado à área de Botânica. Palavras-chave: Herbário, Carajás, Ambiente, Florística.
\end{abstract}

\begin{abstract}
Carajás range, an iron ore area at southeast Pará, inside the Amazon forest had its botany's studies at first related with identification of the types of vegetation found in the area - tropical forest an a kind of shrub savana due to superficial iron ore. With the mine and beneffit path opperating, efforts turned to the research of recovering explored sites, with suitable native species what required the creatioan of an Herbarium avaiable. Also the Herbarium gives suport to research on other subjects and fit Herbarium establishment, february 1987. The Herbarium establishment, february 1987, began with a training program. The Botanic Material has it identification partialy done at Carajás Botanic Park where the Herbarium lays, being sent then to specialists intersted in each botanic family. This work describes the creation of Carajás Herbarium, its aims and contents.
\end{abstract}

Key words: Herbarium, Carajás, Environment, Floristic.

\section{Introdução}

A Serra dos Carajás, localizada no sudeste do Estado do Pará, Município de Parauapebas, tem como produto o minério de ferro explorado pela CVRD. Os estudos botânicos iniciais realizados (Secco \& Mesquita 1983; Silva et al. 1986) se direcionaram para identificação e descrição dos dois tipos de vegetação existentes nos 411 mil hectares do Projeto Ferro Carajás - floresta tropical e campos rupestres e naturais (Conselho Interministerial do Grande Carajás 1981), correlacionados com o afloramento de minério de ferro.

Segundo Silva (1987) a vegetação dos campos rupestres de Carajás apresenta, até então, nove espécies endêmicas e alto grau de especialização. A floresta, com diferenças florísticas locais acentuadas, tem área de intersecção com atividade mineradora de apenas 1 a 2\% da área total do Projeto (Nepomuceno, Com. Pessoal). Com o início da operação da mineração, já a partir de 1986, a pesquisa voltou-se para a recomposição de áreas mineradas, utilizando-se espécies de ocorrência espontânea, o que gerou a necessidade da criação de um herbário acessível para armazenar material botânico e reunir as informações disponíveis.

\footnotetext{
${ }^{1}$ Companhia Vale do Rio Doce - CVRD - Setor de Meio Ambiente - Rua Grajaú, 69 - Núcleo Urbano - 68508 - Carajás - PA.
} 


\section{Material e métodos}

A coleta de material botânico fértil e o registro na caderneta de campo é realizado por equipe do Parque Botânico, treinada segundo metodologia descrita por Mori et al. (1985). Normalmente as plantas são desidratadas no mesmo dia da coleta, não havendo necessidade de tratamentos de preservação.

Para secagem, utiliza-se estufa elétrica e a incorporação das exsicatas no herbário se dá após a identificação do material a nível de família, realizada no Parque Botânico de Carajás.

A determinação de gênero e espécie por um especialista da família é realizada através de doação do material botânico, havendo registros de empréstimos e doações. É utilizado o sistema alfabético para disposição das exsicatas nos armários de aço, sendo utilizada uma sala climatizada através de ar-condicionado e desumidificadores para a conservação do material.

\section{Resultados}

O herbário de Carajás conta atualmente com um acervo de 56 famílias botânicas, distribuídas por amostras herborizadas, a grande maioria identificada a nível de gênero e/ou espećie.

O material botânico coletado até o momento e que consta nos registros do herbário HCJS está discriminado na listagem anexa por ordem alfabética de família, seguida pelo nome científico quando já identificadas e pela inicial do ecossistema de origem: (C) campos rupestres, (T) área de transição natural entre campo e floresta, (F) floresta e (R) áreas de regeneração florestal, constituída principalmente pela margem da Estrada Raymundo Mascarenhas PA-275.

Com exceção da área de transição natural, com apenas $8 \%$, as coletas para o herbário estão distribuídas eqüitativamente pela floresta, regeneração e campo rupestre com respectivamente $35 \%, 29 \%$ e $28 \%$ das exsicatas.

\section{Conclusão}

A formação do herbário de Carajás se constitui em um testemunho da composição florística e subsidiárá à recuperação ambiental, além de prestar suporte à pesquisa de área afins e se enquadrar nos objetivos do Programa de Educação Ambiental, integrantes do currículo escolar de 1\% Grau, implantado na Serra dos Carajás.

Pretende-se expandir esta coleção através das coletas locais sucessivas e aumentar o intercâmbio com as diversas instituições de pesquisas nacionais e internacionais, enviando empréstimo e permuta de material botânico.

\section{Agradecimentos}

Aos funcionários do Parque Botânico de Carajás, Euzamar Cardoso S. Lima e Jair Pereira da Silva, pela dedicação e empenho no preparo das exsicatas. Aos pesquisadores do Museu Paraense Emílio Goeldi, Nélson de Araújo da Rosa e Maria Nazaré C. Bastos, pelo apoio nas determinações. À SUMEI - Superintendência do Meio Ambiente da CVRD- pelo apoio na realização deste trabalho. 


\section{LISTAGEM DO HERBÁRIO DE CARAJÁS - HCJS}

$\begin{array}{ll}\text { Número } & \text { Família } \\ 0046 & \text { ACANTHACEAE } \\ 0070 & \text { ACANTHACEAE } \\ 0082 & \text { ACANTHACEAE } \\ 0118 & \text { ACANTHACEAE } \\ 0120 & \text { ACANTHACEAE } \\ 0130 & \text { ACANTHACEAE } \\ 0141 & \text { AMARANTHACEAE } \\ 0089 & \text { ANACARDIACEAE } \\ 0127 & \text { ANNONACEAE } \\ 0014 & \text { APOCYNACEAE } \\ 0106 & \text { APOCYNACEAE } \\ 0111 & \text { APOCYNACEAE } \\ 0168 & \text { APOCYNACEAE } \\ 0034 & \text { ARALIACEAE }\end{array}$

0051

0019

0028

0050

0072

0083

0090

0103

0104

0121

0126

0147

0155

0145

0080

0132

0129

0002

0016

0035

0063

0125

0112

0001

0015

0025

0030

0048

0088

0040

BIGNONICEAE

BIGNONIACEAE

BIGNONIACEAE

BIGNONIACEAE

BIGNONIACEAE

BIGNONIACEAE

BIGNONIACEAE

BIGNONIACEAE

BIGNONIACEAE

BIGNONIACEAE

BIGNONIACEAE

BIXACEAE

BORAGINACEAE

BROMELIACEAE

COMPOSITAE

COMPOSITAE

COMPOSITAE

COMPOSITAE

COMPOSITAE

CONNARACEAE

CONVOLVULACEAE

CONVOLVULACEAE

CONVOLVULACEAE

CONVOLVULACEAE

CONVOLVULACEAE

CONVOLVULACEAE EUPHORBIACEAE
BIGNONIACEAE

CHRYSOBALANACEAE
Nome Científico

Mendoncia schomburgkiana Nees

-

$-$

-

Ruelia sp.

Tapirira guianensis Aubl

Guatteria villosissima Fries

-

二

Didymopanax morototoni

(Aubl) Done. Planch

Asclepias curasavica

Anemopaegma aff. scabriuscula

-

Amphilophium sp.

Arrabidadea sp.

Tabebuia serratifolia

(Vahl.) Nichols

Frigonacidia sp.

Tabebuia impetiginosa

R

(Mart.) Standl.

Cordia sellowiana Cham

Dickia duckei

Hirtella

Aspilia vandenbergiana J.U.

Santos

-

Vernonia muricata DC.

Conarus perrottetti (DC)

Ipomoea carajasensis. D. Austin

-

$-$

Ipomoea cavalcantei D. Austin

Aparisthimium cordatum (Juss)

Baill
Eco.

$\mathrm{F}$

F

R

F

C

C

R

$\mathrm{T}$

F

C

R

CT

C

F

R

C

$\mathrm{R}$

$\mathrm{T}$

F

C

F

C

R

C

R
R
F
C
R

R
R
F
C
R

.

.

T

T

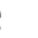

T

F

$\mathrm{C}$
$\mathrm{R}$
$\mathrm{R}$
$\mathrm{C}$
$\mathrm{C}$
$\mathrm{C}$
$\mathrm{F}$

R

C

T

R

C

R

F

C

R

C

C

R

C

R 


\begin{tabular}{|c|c|c|c|}
\hline Número & Família & Nome Científico & Eco. \\
\hline 0079 & EUPHORBIACEAE & Alchorneopsis & $\mathrm{F}$ \\
\hline 0084 & EUPHORBIACEAE & $\begin{array}{l}\text { Mabea angustifolia Spruce ex } \\
\text { Bentham }\end{array}$ & $\mathbf{R}$ \\
\hline 0058 & FLACOURTIACEAE & Casearea grandiflora & $\mathrm{F}$ \\
\hline 0012 & GUTTIFERAE & - & $\mathrm{R}$ \\
\hline 0041 & GUTTIFERAE & - & $\mathrm{F}$ \\
\hline 0151 & GUTTIFERAE & - & $\mathrm{F}$ \\
\hline 0162 & GUTTIFERAE & Rheedia gardneriana $\mathrm{Pl}$ et Tr. & $\mathrm{F}$ \\
\hline 0054 & ICACINACEAE & Emmotum sp. & $\mathrm{C}$ \\
\hline 0128 & LACISTEMACEAE & Lácistema pubescens Mart & $\mathrm{F}$ \\
\hline 0031 & LAURACEAE & Nectandra cuspidata Ness & $\mathrm{F}$ \\
\hline 0032 & LAURACEAE & - & $\mathrm{F}$ \\
\hline 0033 & LAURACEAE & - & $\mathrm{F}$ \\
\hline 0066 & LAURACEAE & Mezilaurus sp. & $\mathrm{T}$ \\
\hline 0077 & LAURACEAE & Mezilaurus sp. & $\mathrm{F}$ \\
\hline 0087 & LAURACEAE & Nectandra sp. & $\mathrm{F}$ \\
\hline 0027 & LECYTHIDACEAE & - & $\mathrm{F}$ \\
\hline 0045 & LECYTHIDACEAE & Couratari guianensis & $\mathrm{F}$ \\
\hline 0150 & LECYTHIDACEAE & Lecythis lurida (Miers) Mori & $\mathrm{F}$ \\
\hline 0160 & LECYTHIDACEAE & $\begin{array}{l}\text { Lecythis pisonis subsp. usitata } \\
\text { (Miers.) Mori \& Prance }\end{array}$ & $\mathrm{F}$ \\
\hline 0005 & LEGUMINOSAE 149 & $\begin{array}{l}\text { Mimosa acutistipula var. } \\
\text { ferrea Barneby }\end{array}$ & $\mathrm{C}$ \\
\hline 0009 & LEGUMINOSAE 149 & - & $\mathrm{T}$ \\
\hline 0036 & LEGUMINOSAE 149 & Inga cinnamomea Spruce & $\mathrm{F}$ \\
\hline 0042 & LEGUMINOSAE 149 & - & $\mathrm{F}$ \\
\hline 0043 & LEGUMINOSAE 149 & Inga ef marginata & $\mathrm{F}$ \\
\hline 0049 & LEGUMINOSAE 149 & $\begin{array}{l}\text { Mimosa somnians HBK Var. visc. } \\
\text { (W.) Barneby }\end{array}$ & $\mathrm{C}$ \\
\hline 0064 & LEGUMINOSAE 149 & - & $\mathrm{F}$ \\
\hline 0096 & LEGUMINOSAE 149 & Parkia pendula Benth. & TF \\
\hline 0102 & LEGUMINOSAE 149 & - & $\mathrm{R}$ \\
\hline 0113 & LEGUMINOSAE 149 & Newtonia suaveolens (Miquel.) Breran & $\mathrm{F}$ \\
\hline 0165 & LEGUMINOSAE 149 & - & $\mathrm{F}$ \\
\hline 0004 & LEGUMINOSAE 150 & - & $\mathrm{R}$ \\
\hline 0007 & LEGUMINOSAE 150 & Bauhinia pulchella Benth & $\mathrm{C}$ \\
\hline 0024 & LEGUMINOSAE 150 & $\begin{array}{l}\text { Chamaecrista flexuosa L. var. } \\
\text { flexuosa }\end{array}$ & $\mathrm{R}$ \\
\hline 0095 & LEGUMINOSAE 150 & - & $\mathrm{R}$ \\
\hline 0098 & LEGUMINOSAE 150 & - & $\mathrm{R}$ \\
\hline 0114 & LEGUMINOSAE 150 & Chamaecrista bahieae Irwin & $\mathrm{F}$ \\
\hline 0124 & LEGUMINOSAE 150 & - & $\mathrm{R}$ \\
\hline 0133 & LEGUMINOSAE 150 & - & $\mathrm{R}$ \\
\hline 0142 & LEGUMINOSAE 150 & - & $\mathrm{R}$ \\
\hline 0164 & LEGUMINOSAE 150 & Sclērolobium paraensis & TC \\
\hline 0022 & LEGUMINOSAE 152 & Periandra sp. & $\mathrm{C}$ \\
\hline 0044 & LEGUMINOSAE 152 & - & $\mathrm{F}$ \\
\hline 0060 & LEGUMINOSAE 152 & Stylosanthes sp. & $\mathrm{R}$ \\
\hline
\end{tabular}




\begin{tabular}{|c|c|c|}
\hline Número & Família & Nome Científico \\
\hline 0065 & LEGUMINOSAE 152 & - \\
\hline 0091 & LEGUMINOSAE 152 & Dioclea virgata (Rich.) Amsh. \\
\hline 0135 & LEGUMINOSAE 152 & - \\
\hline 0158 & LEGUMINOSAE 152 & - \\
\hline 0163 & LEGUMINOSAE 152 & - \\
\hline 0169 & LEGUMINOSAE 152 & Poecilanthe effusa Ducke \\
\hline 0085 & LORANTHACEAE & Phthirusa paniculata (HBK) \\
\hline 0003 & LYTRACEAE & Cuphea annulata Koelm \\
\hline 0100 & LYTRACEAE & Cuphea tenella Koelm \\
\hline 0008 & MALPIGHIACEAE & Byrsonima coriaceae (SW) Kunth \\
\hline 0018 & MALPIGHIACEAE & Byrsonima sp. \\
\hline 0020 & MALPIGHIACEAE & - \\
\hline 0061 & MALPIGHIACEAE & - \\
\hline 0069 & MALPIGHIACEAE & - \\
\hline 0107 & MALPIGHIACEAE & - \\
\hline 0122 & MALPIGHIACEAE & - \\
\hline 0140 & MALPIGHIACEAE & - \\
\hline 0144 & MALPIGHIACEAE & - \\
\hline 0146 & MALPIGHIACEAE & - \\
\hline 0149 & MALPIGHIACEAE & - \\
\hline 0006 & MALVACEAE & - \\
\hline 0023 & MALVACEAE & - \\
\hline 0039 & MALVACEAE & - \\
\hline 0093 & MALVACEAE & - \\
\hline 0097 & MALVACEAE & Hibiscus megalofila \\
\hline 0143 & MARCGRAVIACEAE & Norantea sp. \\
\hline 0017 & MELASTOMATACEAE & - \\
\hline 0021 & MELASTOMATACEAE & - \\
\hline 0029 & MELASTOMATACEAE & - \\
\hline 0047 & MELASTOMATACEAE & - \\
\hline 0062 & MELASTOMATACEAE & - \\
\hline 0101 & MELASTOMATACEAE & - \\
\hline 0116 & MELASTOMATACEAE & - \\
\hline 0161 & MELIACEAE & Guarea kunthiana Juss. \\
\hline 0154 & MENISPERMACEAE & $\begin{array}{l}\text { Abuta grandiflora (Mart.) } \\
\text { Sandwith }\end{array}$ \\
\hline 0157 & MORACEAE & $\begin{array}{l}\text { Clarisia ilicifolia (Spreng) } \\
\text { Lanj. et. Rossb }\end{array}$ \\
\hline 0038 & MYRISTICACEAE & - \\
\hline 0057 & MYRISTICACEAE & Campsoneura sp. \\
\hline 0073 & MYRTACEAE & - \\
\hline 0105 & MYRTACEAE & - \\
\hline 0117 & MYRTACEAE & Eugenia sp. \\
\hline 0134 & OCHNACEAE & Ouratea castanaefolia \\
\hline 0099 & OLACACEAE & Heisteria sp. \\
\hline 0092 & ONAGRACEAE & Ludwigia sp. \\
\hline 0138 & ORCHIDACEAE & Sobralia liliastrum Lindl. \\
\hline 0013 & PASSIFLORACEAE & - \\
\hline 0108 & PASSIFLORACEAE & Passiflora coccinea Aubl. \\
\hline
\end{tabular}




\begin{tabular}{|c|c|c|}
\hline Número & Família & Nome Científico \\
\hline 0153 & PASSIFLORACEAE & - \\
\hline 0166 & PASSIFLORACEAE & \\
\hline 0074 & PHYTOLACCACEAE & Phytolacea sp. \\
\hline 0159 & PHYTOLACCACEAE & - \\
\hline 0123 & PIPERACEAE & - \\
\hline 0156 & PIPERACEAE & - \\
\hline 0052 & POLYGALACEAE & Polygala sp. \\
\hline 0053 & PROTEACEAE & - \\
\hline 0067 & RHAMNACEAE & Gouania pyrifolia Reiss \\
\hline 0059 & RUBIACEAE & Perama carajasensis Kirkbride \\
\hline 0110 & RUBIACEAE & Borreria verticilata \\
\hline 0055 & RUTACEAE & Pilocarpus microphyllus Stapf. \\
\hline 0071 & RUTACEAE & Zanthoxylum sp. \\
\hline 0078 & RUTACEAE & Pilocarpus itacaiunensis Pires \\
\hline 0056 & SAPINDACEAE & Matayba sp. \\
\hline 0086 & SAPINDACEAE & Matayba arborencens (Aubl.) Radlk \\
\hline 0011 & SAPOTACEAE & - \\
\hline 0115 & SAPOTACEAE & Richardella manausensis Aubl. \\
\hline 0026 & SOLANACEAE & - \\
\hline 0037 & SOLANACEAE & - \\
\hline 0075 & SOLANACEAE & Solanum sp. \\
\hline 0081 & SOLANACEAE & Solanum sp. \\
\hline 0094 & SOLAIIACEAE & - \\
\hline 0109 & SOLANACEAE & - \\
\hline 0131 & SOLANACEAE & - \\
\hline 0152 & SOLANACEAE & - \\
\hline 0137 & STERCULIACEAE & $\begin{array}{l}\text { Theobroma grandiflorum } \\
\text { (Willd. ex Spreng.) Schum. }\end{array}$ \\
\hline 0136 & TILIACEAE & - \\
\hline 0148 & TURNERACEAE & Turnera sp. \\
\hline 0170 & VELLOZIACEAE & - \\
\hline 0010 & VERBENACEAE & Amasonia campestris \\
\hline 0076 & VERBENACEAE & Aegiphila sp. \\
\hline 0167 & VERBENACEAE & - \\
\hline 0068 & VITACEAE & Cissus erosa Rich. \\
\hline 0139 & VOCHYSIACEAE & Vochysia maxima Ducke \\
\hline
\end{tabular}

Referências Bibliográficas

CONSELHO INTERMINISTERIAL DO PROGRAMA GRANDE CARAJÁS. 1981. Aspecto físicos, demográficos e fundiários. Rio. de Janeiro.

MORI, S.A.; SILVA, L.A.M.; LISBOA, G. \& CORADIN, L. 1985. Manual de Manejo do Herbário Fanerogâmico. Salvador, CEPLAC-BA.

SECCO, R. de S. \& MESQUITA, A.L. 1983. Notas sobre a vegetação de canga da Serra Norte - I. Bol. Mus. Para. Emílio Goeldi. Nova Ser. Bot., Belém, (59): 1-13; Jan. il. 
SILVA, M.F.F. da; MENEZES, N.L. de; CAVALCANTE, P.B. \& JOLY, C.A. 1986. Estudos botânicos: histórico, atualidade e perspectivas. In: CARAJÁS: desafio político, ecologia e desenvolvimento. São Paulo, Brasiliense; Brasília, CNPq. p.184-207. SILVA, M.F.F. da. 1987. Estudos botânicos em Carajás. In: SEMINÁRIO SOBRE DESENVOLVIMENTO ECONÔMICO E IMPACTO AMBIENTAL EM ÁREAS DE TRÓPICO ÚMIDO BRASILEIRO A EXPERIÊNCIA DA CVRD. 1. Belém, 1986. Anais... Rio de Janeiro, CVRD. p. 93-9. 